\title{
La resolución de problemas, una oportunidad para aprender
} Intervención pedagógica en Matemáticas

Problem solving an opportunity to learn: Pedagogical intervention in Mathematics

Daissy Lorena Rodríguez Ariza Alleidy Rodriguez Ariza

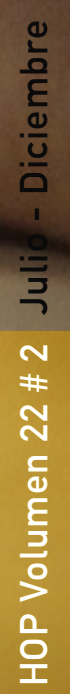

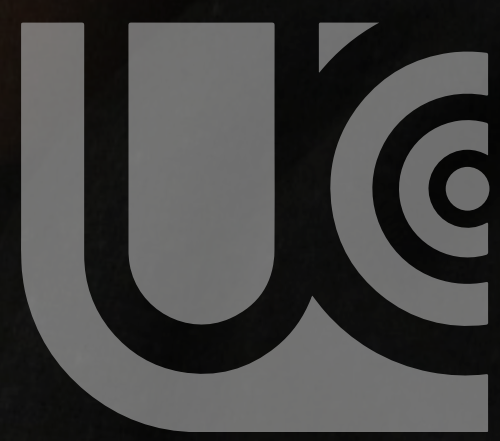

HORIZONTES PEDAGÓGICOS ISSN-I: 0123-8264 | e-ISSN: 2500-705X 
PEDAGÓGICOS

ID:

0123-8264.hop.22205

Title: $\quad$ Problem solving an opportunity to learn

Subtitle: Pedagogical intervention in mathematics

Título: $\quad$ La resolución de problemas, una oportunidad para aprender

Subtítulo: Intervención pedagógica en matemáticas

Alt Title / Título alternativo:

[en]:

Problem solving an opportunity to learn

Author (s) / Autor (es):

Rodríguez Ariza \& Rodriguez Ariza

Keywords / Palabras Clave:

[en]:

Problem based learning; primary school mathematics; intervention; classroom research; mathematics teachers; school wisdom and practices; student progress

[es]: Aprendizaje basado en problemas; matemáticas en primaria; intervención; investigación en el aula; profesores de matemáticas; saberes y practicas escolares; progreso estudiantil.

Submited: 2020-04-13

Acepted: 2020-04-22

\section{Resumen}

El propósito de este artículo producto de investigación, es dar a conocer los alcances de un estudio correlacional realizado entre Junio del 2017 y noviembre de 2018 en dos instituciones públicas (Bogotá D.C y Soacha, Cundinamarca), cuyo objetivo era potencializar las competencias matemáticas para la resolución de problemas a través de la implementación de una unidad didáctica constructivista en los estudiantes de quinto grado de educación básica de la Institución Educativa Nuevo Compartir y la I.E.D. Darío

Echandía; para esto, se empleó la observación participante de los estudiantes, sus reportes académicos en el área de matemáticas y se tomaron los resultados de la "Prueba Saber"

(2016) y los resultados de la prueba "Aprendamos" (2017), analizándolos desde tres categorías: comunicación, razonamiento y resolución; donde se evidenció la dificultad que tenían los estudiantes al solucionar problemas matemáticos. El trabajo investigativo se rigió bajo los parámetros del paradigma hermenéutico, con enfoque mixto, los referentes teóricos se plantean desde el concepto de competencia matemática para el Ministerio de Educación Nacional y de diferentes autores hasta la concepción de una unidad didáctica constructivista. Las técnicas empleadas

para la recolección de la información fueron la observación participante, el análisis documental,

el taller investigativo, grupos focales y grupos

de discusión. Como resultado se destaca el progreso significativo de los estudiantes en cada una de las competencias matemáticas, siendo el razonamiento la categoría con más avance durante la intervención en ambas entidades escolares. Esta evolución se ve representada en los estudiantes al leer detenidamente los enunciados, identificar los datos, interpretar en forma asertiva los enunciados

de problemas matemáticos y preguntar por los

términos desconocidos. Como valor agregado

al producto del trabajo investigativo, se debe incluir el fomento de habilidades sociales como la capacidad de escucha, compartir una opinión y la empatía al trabajar por grupos colaborativos, demostrándose así la pertinencia y eficiencia de la propuesta pedagógica.

\section{Abstract}

The purpose of this research product article is to present the scope of a correlational study carried out between June 2017 and November 2018 in two public institutions (Bogotá DC and Soacha, Cundinamarca), whose objective was to potentiate mathematical competences for the problem solving, through the implementation of a constructivist didactic unit in the fifth grade students of basic education of the Institución

Educativa Nuevo Compartir and the Darío Echandía IED College, for this the participant observation of the students was used, their academic reports in the area of mathematics and the results of the Prueba Saber (2016) and Aprendamos (2017) were taken, analyzing them from three categories: communication, reasoning and resolution; where the difficulty that the students had in solving mathematical problems was evident. The investigative work

was governed under the parameters of the hermeneutic paradigm, with a mixed approach, the theoretical references arise from the concept of mathematical competence for the Ministry of

National Education and of different authors to the conception of a constructivist didactic unit. The techniques used to collect the information are participant observation, documentary analysis, research workshop, focus and discussion groups. As results, the significant progress of students in each of the mathematical competences stands out, reasoning being the category with the most progress during the intervention in both school entities. This evolution is represented in students by carefully reading the statements, identifying the data, assertively interpreting the statements of mathematical problems and asking for unknown

terms. As an added value to the product of the research work, the promotion of social skills such as listening skills, sharing an opinion and empathy when working in collaborative groups should be included, thus demonstrating the relevance and efficiency of the pedagogical proposal.

\section{Citar como:}

Rodríguez Ariza, D. L. \& Rodriguez Ariza, A. . (2020). La resolución de problemas, una oportunidad para aprender: Intervención pedagógica en matemáticas. Horizontes Pedagógicos, 22 (2), 37-48. Obtenido de: https://horizontespedagogicos.ibero.edu.co/article/view/1826

Msc Daissy Lorena Rodríguez Ariza

ORCID: $\quad$ https://orcid.org/0000-0002-7090-

9426

\section{Source I Filiacion:}

Universidad Cooperativa de Colombia[co]

BIO:

Magister en Dificultades del Aprendizaje, Especialista en el Aprendizaje Escolary sus dificultades, Licenciada en Lengua Castellana y Comunicación.

City I Ciudad:

Bogotá DC [CO]

e-mail:

daissy.rodrigueza.@campusucc.edu.co
Msc Alleidy Rodriguez Ariza, Lic

ORCID: $\quad$ https://orcid.org/0000-0002-1351-

4591

\section{Source I Filiacion}

Universidad Cooperativa de Colombia[co] BIO:

Magister en dificultades del aprendizaje, especialista en aprendizaje escolary sus dificultades, Licenciada en lengua castellana y comunicación City I Ciudad:

Bogotá DC [CO]

e-mail:

alleidy.rodrigueza@campusucc.edu.co 


\section{La resolución de problemas, una oportunidad para aprender Intervención pedagógica en Matemáticas}

Problem solving an opportunity to learn: Pedagogical intervention in Mathematics Daissy Lorena Rodríguez Ariza Alleidy Rodriguez Ariza

\section{Introducción}

En la investigación se abordó el concepto de competencia, competencia matemática y resolución de problemas matemáticos desde la perspectiva y posturas de diferentes autores, con el fin de establecer criterios para el diseño e implementación de la unidad didáctica.

El trabajo por competencias es hoy en día, un reto para el sistema educativo nacional Colombiano, de ahí la importancia de conocer sus implicaciones pedagógicas; paraZabala (2007), una competencia es el conjunto deconocimientos que necesita conocer cualquier persona para dar respuesta a los problemas que enfrenta a lo largo de su vida. Perrenoud (2001), define la competencia como la aptitud para enfrentar eficazmente una familia de situaciones análogas movilizando a conciencia una variedad de recursos cognitivos. Estas definiciones tienen en común considerar la competencia como la capacidad de dar respuesta a las diferentes situaciones que se le presentan, lo que se conoce como el saber actuar en contexto. En esa misma línea, se encuentra la definición hecha por el Ministerio de Educación (MEN) en los estándares Básicos de Matemáticas (2006), quien la define como el conjunto de conocimientos que le permiten al individuo actuar en contextos nuevos y retadores; es decir, es el conjunto de habilidades y conocimientos que facultan al estudiante para actuar en una situación real, dándole sentido y significado al proceso de enseñanza. 
La resolución de problemas, una oportunidad para aprender Intervención pedagógica en matemáticas

Hezkuntza (2000), plantea que el trabajo porcompetencias necesita de un aprendizaje activo, que prepare al estudiante para saber ser, para saber hacer y para saber aplicar el conocimiento en una situación específica; convirtiéndose en un gran reto para la escuela, quien debe disponer de ambientes que permitan recrear situaciones donde el estudiante ponga en práctica lo aprendido. Zabala (2007), sostiene que las estrategias empleadas en la enseñanza por competencias deben provocar un conflicto cognitivo y promover una actividad mental necesaria para establecer relaciones entre los contenidos nuevos y los conocimientos previos; factores determinantes en el diseño de la propuesta de esta investigación.

Para el MEN (2006), un estudiante es competente en matemáticas cuando está en la capacidad de utilizar el saber matemático para resolver problemas, comprender y argumentar los procesos empleados en su resolución. En ese mismo documento se plantean cinco competencias: la ejercitación de procedimientos, relacionada con el uso de los procedimientos matemáticos o algoritmos de forma rápida y eficaz. La modelación, referida a la capacidad que tienen los estudiantes para relacionar las matemáticas con el contexto, creando modelos que le permitan resolver problemas.

La Comunicación que implica conocer el lenguaje propio del área; el razonamiento definido como la capacidad para analizar, argumentar y planear una estrategia que dé solución a una situación matemática y, por último, la resolución de problemas donde están implícitas las demás competencias, porque es la capacidad que tiene el estudiante para enfrentarse a problemas matemáticos no rutinarios que requieren de nuevos modelos para resolverse.

Siendo la resolución de problemas una competencia que necesita de las demás para desarrollarse, se convierte en uno de los objetivos del área de matemáticas. Autores como Guzmán (1989), Pozo (1995) y Campistrous (1996), señalan la resolución de problemas como el corazón de la enseñanza de las matemáticas, porque responde al orden natural de las cosas: el conocimiento debe ser siempre la respuesta a una pregunta previamente formulada.

Por su parte, Polya (1965), sostiene que resolver problemas es una cuestión de habilidad práctica, para lo cual se debe ejercitar al estudiante para ello, por lo tanto, la tarea del docente consistirá en proporcionarle el mayor número de ocasiones de imitación y práctica; además de implementar las siguientes cuatro fases: comprender el problema, concebir el plan, ejecutarlo y hacer una visión retrospectiva sobre el proceso realizado.

\section{Metodología}

Para el análisis e interpretación de la información, el proyecto de investigación se rigió bajo los parámetros del paradigma Hermenéutico, porque se interpretó el contexto educativo de los estudiantes del curso 503 de la Institución Educativa Nuevo Compartir y 502 de la I.E.D Colegio Darío Echandía; especialmente su desempeño en el área de matemáticas, a través del análisis inductivo; se toman datos específicos de las pruebas externas (Saber y Aprendamos) e información interna (los reportes académicos finales del área de matemáticas) para construir conclusiones generales sobre la habilidad que tienen los estudiantes en la resolución de problemas. Además, el equipo investigador estaba relacionado con el objeto de estudio, convirtiéndose en una herramienta directa para obtener información sobre la problemática presentada. a través de dos técnicas; observación participante (Corbetta, 2007), que se realiza a través de una lista de chequeo con base a las categorías de análisis y análisis documental (Corbetta, 2007), en la que se hace una interpretación del Reporte de Excelencia 2017, antecedentes de investigación y fuentes bibliográficas.

\section{Resultados: Técnicas de Investigación.}

En ese sentido, para abordar la descripción de la dificultad que tenían los estudiantes del curso 503 de la Institución Educativa Nuevo Compartir al solucionar problemas matemáticos, se hizo un recorrido de los principios institucionales, los resultados de la Prueba Saber en el área de matemáticas que presentó el curso en el año 2015, los resultados de la Prueba Aprendamos presentada en el año 2017 y el reporte académico final del área de matemáticas del año 2017.

En concordancia con el MEN, el trabajo en matemáticas sobre la resolución de problemas es también la preocupación del Proyecto Educativo Institucional (2017), en el cual plantea como uno de los principios institucionales la formación de niños y jóvenes capaces de participar en la solución de problemas de su comunidad, propiciando estrategias de productividad laboral en el ámbito local y global.

De ahí, que la Institución Educativa ha mostrado especial preocupación por mejorar el índice sintético de calidad (ISCE), estableciendo a través del consejo académico la aplicación de las pruebas bimestrales al finalizar cada periodo lectivo, elaboradas por una entidad externa. Sin embargo, los resultados obtenidos no son los esperados, porque el ISCE para el año 2017, bajó cuatro centésimas, paso del 4,24 al 4,20 en comparación con el año 2016.

Al comparar los resultados del 2015 con los del 2016 en el área de matemáticas para grado tercero de la Institución Educativa Nuevo Compartir, recalcando que el curso 503 presento la prueba en el 2016, se puede señalar las siguientes interpretaciones, a partir de la figura 1:

Figura 1 Resultados Prueba Saber 2015 vs 2016

\section{PROGRESO}

PORCENTAJE DE ESTUDIANTES EN NIVEL INSUFICIENTE La escala de valores es de 0 a $100 \%$

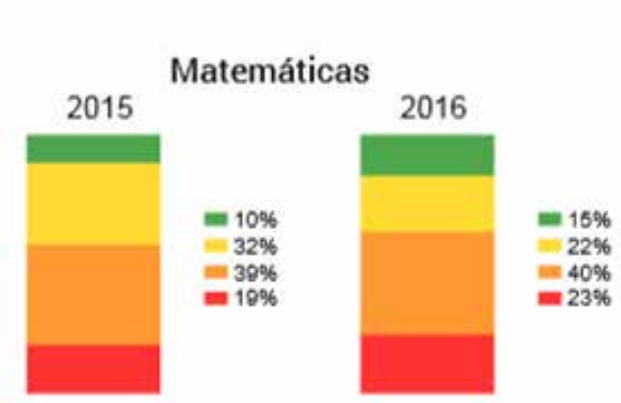

Tercero

Fuente: Gráfica tomada del reporte de excelencia (ICFES, 2017).

$\rightarrow$ El nivel de insuficiente aumentó el 4\%, pasó del 19\% al 23\%

$\rightarrow$ El nivel mínimo aumentó el 1\%, pasó del 39\% al 40\%.

$\rightarrow$ El nivel satisfactorio bajó el 10\%, pasó del 32\% al 22\%.

$\rightarrow$ El nivel avanzado aumentó el 5\%, pasó del 10\% al 15\%. 
De esta manera se aprecia que un 63\% de los estudiantes se encontraban en un nivel insuficiente y mínimo, y el puntaje promedio para la prueba de matemáticas en grado tercero de la Institución es de 293 en comparación con el de Soacha que es de 327, ubicando a la Institución en el puesto 134 entre 155 instituciones públicas y privadas. Un puesto muy bajo en la escala, que deja en evidencia las falencias en las prácticas pedagógicas que se llevan a cabo en el área de matemáticas.

Los resultados de la prueba Aprendamos confirman los resultados de las pruebas Saber. Estas fueron diseñadas por el MEN como estrategia para la evaluación formativa de los estudiantes de segundo y cuarto grado de básica primaria en las áreas de lenguaje y matemáticas de los establecimientos públicos del país. La prueba fue aplicada en forma Online a los estudiantes del curso 503 cuando estaban cursando el grado cuarto, bajo el acompañamiento del director de curso, el 17 de abril de 2017. La prueba mide para el área de matemáticas el nivel en las tres competencias básicas y estos son los porcentajes de estudiantes que presentaban dificultades en comparación con los resultados de la prueba Saber 2016, tal como se puede apreciar en la tabla 1:

Tabla 1 Resultados Prueba Aprendamos y Prueba Saber, Institución Educativa Nuevo Compartir.

$\begin{array}{cccc}\begin{array}{c}\text { Compe- } \\ \text { tencia } \\ \text { Prueba }\end{array} & \begin{array}{c}\text { comuni- } \\ \text { cación }\end{array} & \begin{array}{c}\text { Razona- } \\ \text { miento }\end{array} & \begin{array}{l}\text { Resolu - } \\ \text { ción }\end{array} \\ \begin{array}{c}\text { Saber } \\ \text { Apren - }\end{array} & 73 \% & 74 \% & 71 \% \\ \text { damos } & 62 \% & 63 \% & 63 \%\end{array}$

Fuente: Elaboración propia basada en los reportes de la Prueba Aprendamos 2017 y Saber 2016.

Al comparar la Prueba Saber 2016 y Aprendamos 2017 se evidencia la relación y correspondencia de los resultados obtenidos por la misma población en diferentes años, donde los porcentajes de dificultad presentados en cada una de las competencias matemáticas siguen siendo altos.

Esta situación se veía reflejada en el trabajo en aula, ya que los estudiantes del curso 503 presentaban dificultades en la solución de problemas matemáticos con números naturales, ellos conocen y ejecutan los algoritmos de las operaciones básicas (adición, sustracción, multiplicación y división), pero se les dificultaba su aplicación en un contexto.

Ante un problema matemático, los estudiantes buscaban la ayuda del docente o la de un compañero, empezaban a observar el cuaderno de los demás, preguntando qué operación debe realizarse, dedicaban poco tiempo a la lectura del enunciado, no identifican los datos, descartan las posibles acciones a realizar: "¿hay que sumar?, ¿hay que restar?". Se muestran bastante inquietos durante la actividad parándose del puesto varias veces, piden permiso para ir al baño o solicitan prestados los útiles escolares. Cuando se les planteaba un problema matemático que requieren de dos o más operaciones para resolverse, por lo general realizaban sólo un algoritmo. En cambio, si se les propone crear el problema matemático a partir de una operación dada, un 75\% lograba el objetivo.

Además, desconocían terminología clave para la interpretación del enunciado, por ejemplo, la diferencia, el factor, el triple, la cuarta parte, entre otros. Al darle respuesta a los problemas se remitían a colocar el número sin relacionarlo con el contexto enunciado. Presentaban apatía por las actividades de clase donde debían solucionar problemas matemáticos, demostrando predisposición; esta información es recolectada a través de la observación participante, consignada en la lista de chequeo.

El reporteacadémico final para el año 2017 delárea de matemáticas cuando el curso 503 se encontraba en grado cuarto, da cuenta del desempeño básico, tal y como se puede apreciar en la figura 2:

Figura 2 Reporte Académico final, curso 403, Institución Educativa Nuevo Compartir

Reporte académico final 2017

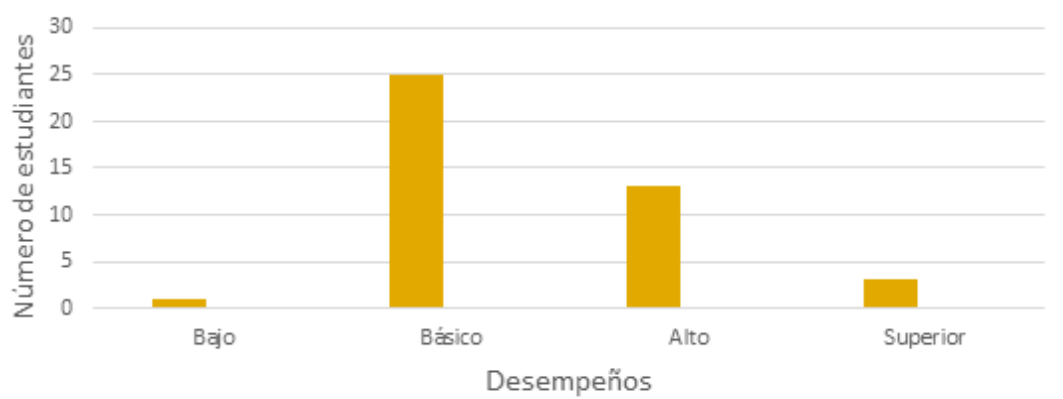

Fuente: Elaboración propia a partir del informe académico para el área de matemáticas del curso 403.

Para describir la dificultad que tienen los estudiantes del curso 502 de la I.E.D. Colegio Darío Echandía; en la solución de problemas matemáticos, se hace referencia a los principios institucionales, se analizan los resultados de la Prueba Saber 2016 cuando el curso 502 cursaba grado tercero, el comparativo del Índice Sintético de Claridad 2016- 2017, el reporte académico final del año 2017 para el área de matemáticas; se aclara que la institución no presenta resultados de la Prueba Aprendamos porqué sólo se realiza a las instituciones que se encuentran dentro del Proyecto Todos a Aprender.

Respecto al el Proyecto Educativo Institucional (2017), el colegio plantea como uno de los principios institucionales la formación integral de ciudadanos con valores sociales, capaces de solucionar los problemas de su entorno y participar activamente en la construcción de una sociedad solidaria y justa. Demostrando así un interés por una educación donde el estudiante pueda poner en contexto todos los aprendizajes adquiridos, orientándolos hacia el servicio de la comunidad.

Para tal fin se ha dispuesto a través del consejo académico la implementación de pruebas bimestrales por cada periodo, donde se aborda la solución de problemas desde todas las áreas, con el propósito de mejorar el índice Sintético de Calidad, pero pese a los esfuerzos este puntaje ha ido bajando, para el 2016 era de 5,83 y para el 2017 de 5,80.

En la figura 3 se hace una comparación de los resultados de la Prueba Saber 2015 con los del 2016 para el área matemáticas, cuando los estudiantes del 502 cursaban grado tercero. Figura 3 Resultados Prueba Saber 2015 vs 2016, Colegio Darío
Echandía I.E.D

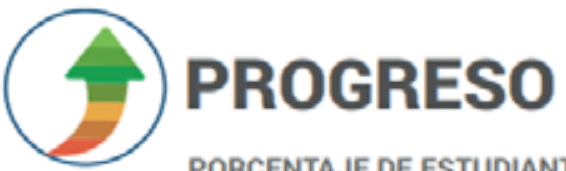

PORCENTAJE DE ESTUDIANTES EN NIVEL INSUFICIENTE La escala de valores es de 0 a $100 \%$

Tercero

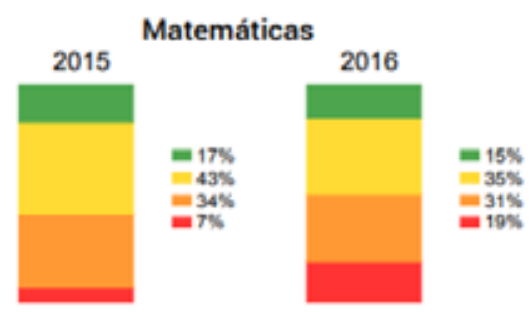


La resolución de problemas, una oportunidad para aprender Intervención pedagógica en matemáticas

Fuente: Gráfica tomada del reporte de excelencia (ICFES, 2017)

A partir de la figura 3 se puede señalar:

$\rightarrow$ El nivel insuficiente aumentó el 12\%, pasó del 7\% al 19\%|

$\rightarrow$ El nivel mínimo bajó el 3\%, pasó del 34\% al 31\%.

$\rightarrow$ El nivel satisfactorio bajó el 8\%, pasó del 43\% al 35\%.

$\rightarrow$ El nivel avanzado bajó el 2\%, pasó del 17\% al 15\%.

Es preocupante el aumento de la población con un desempeño insuficiente en la prueba, representado en un 12\% en comparación con el año 2015, ubicando a la institución en el puesto 534 entre instituciones públicas y privadas de Bogotá. En cuanto al reporte académico final del área de matemáticas del año 2017, cuando los estudiantes cursaban grado cuarto, se destaca el desempeño básico de los estudiantes, como se muestra en la figura 4:

Figura 4 Reporte académico final en matemáticas, curso 402, Colegio Darío Echandía I.E.D

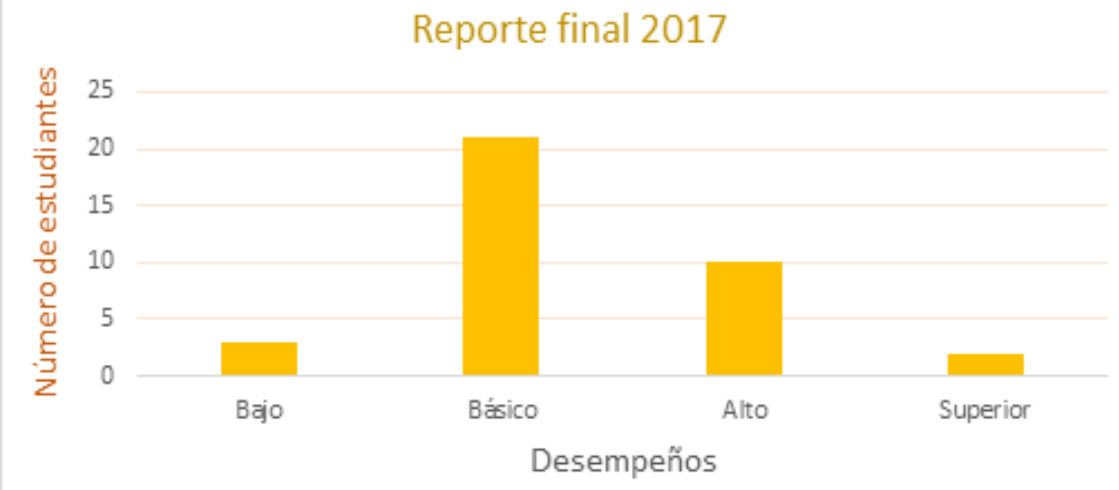

Fuente: elaboración propia a partir del informe académico para el área de matemáticas del Colegio Darío Echandía I.E.D

El porcentaje de estudiantes con desempeño bajo y básico en el área de matemáticas es de 66\%, un porcentaje alto que deja a tres estudiantes sin promoción a grado quinto.

A través de la observación participante se detecta que los estudiantes se mostraban ansiosos durante la resolución de problemas, evidenciado levantarse varias veces del puesto, miraban los cuadernos de los demás, pasaban las hojas de los cuadernos hacia adelante y hacia atrás, abrían y cerraban la maleta, cambiaban la cartuchera de puesto, pedían permiso para ir al baño frecuentemente y se quitaban la chaqueta del uniforme. Así mismo, las preguntas que hacian al docente estaban orientadas al algoritmo que debía realizarse, "Profesora, ¿Sumamos o restamos?", ¿Qué hay que hacer profesora?", "¿Qué nos están preguntando?”; además dedicaban poco tiempo a la lectura de los enunciados y la construcción de la respuesta limitándose a colocar el resultado de la operación matemática sin hacer referencia al contexto del enunciado.

A continuación, se hace la comparación entre los resultados de la Prueba Saber 2016 y los indicadores evaluados en las tres competencias matemáticas (comunicación, razonamiento y resolución) de las dos instituciones, a través de las tablas 2 y 3

Tabla 2 Resultados Prueba Saber 2016, cuadro comparativo entre las dos instituciones educativas

$\begin{gathered}\text { Competencia } \\ \text { Institución ción }\end{gathered}$
$\begin{aligned} & \text { Institución Edu- } \\ & \text { cativa Nuevo Com- }\end{aligned}$
$\begin{aligned} & \text { Nartir } \\ & \text { Colegio Darío }\end{aligned}$
$\begin{aligned} & \text { Echandía l.E.D } \\ & \text { miento }\end{aligned}$

Fuente: Elaboración propia a partir del Reporte de Excelencia (ICFES, 2017). Tabla 3 Resultados para grado tercero de la Prueba Saber, cuadro comparativo por indicadores de competencias matemáticas entre las dos instituciones educativas.

\begin{tabular}{|c|c|c|c|}
\hline \multirow[b]{2}{*}{$\begin{array}{l}\text { Compe- } \\
\text { tencia }\end{array}$} & \multirow[b]{2}{*}{ Indicador } & \multicolumn{2}{|c|}{$\begin{array}{l}\text { Población que presenta } \\
\text { dificultad }\end{array}$} \\
\hline & & $\begin{array}{l}\text { Institu- } \\
\text { ción Educativa } \\
\text { Nuevo Com- } \\
\text { partir }\end{array}$ & $\begin{array}{l}\text { Colegio } \\
\text { Darío Echan- } \\
\text { día I.E.D }\end{array}$ \\
\hline \multirow[t]{5}{*}{$\begin{array}{l}\text { Comuni- } \\
\text { cación }\end{array}$} & $\begin{array}{l}\text { Describe secuencias nu- } \\
\text { méricas y geométricas }\end{array}$ & $66 \%$ & $62 \%$ \\
\hline & $\begin{array}{l}\text { Reconoce equivalencias } \\
\text { entre diferentes tipos de repre- } \\
\text { sentación relacionadas con } \\
\text { números. }\end{array}$ & $59 \%$ & $49 \%$ \\
\hline & $\begin{array}{l}\text { Establece correspon- } \\
\text { dencia entre objetos, eventos, } \\
\text { patrones o instrumentos de } \\
\text { medida }\end{array}$ & $55 \%$ & $50 \%$ \\
\hline & $\begin{array}{l}\text { Ubica objetos con base } \\
\text { a instrucciones referentes a } \\
\text { dirección, distancia y posición. }\end{array}$ & $54 \%$ & $56 \%$ \\
\hline & $\begin{array}{l}\text { Clasifica y organiza da- } \\
\text { tos. }\end{array}$ & $50 \%$ & $49 \%$ \\
\hline & $\begin{array}{l}\text { Usa operaciones y pro- } \\
\text { piedades de los números para } \\
\text { establecer relaciones entre } \\
\text { ellos en situaciones específi- } \\
\text { cas. }\end{array}$ & $81 \%$ & $72 \%$ \\
\hline & $\begin{array}{l}\text { Establece conjeturas } \\
\text { que se aproximan a las nocio- } \\
\text { nes de paralelismo y perpendi- } \\
\text { cularidad en figuras planas. }\end{array}$ & $76 \%$ & $74 \%$ \\
\hline
\end{tabular}

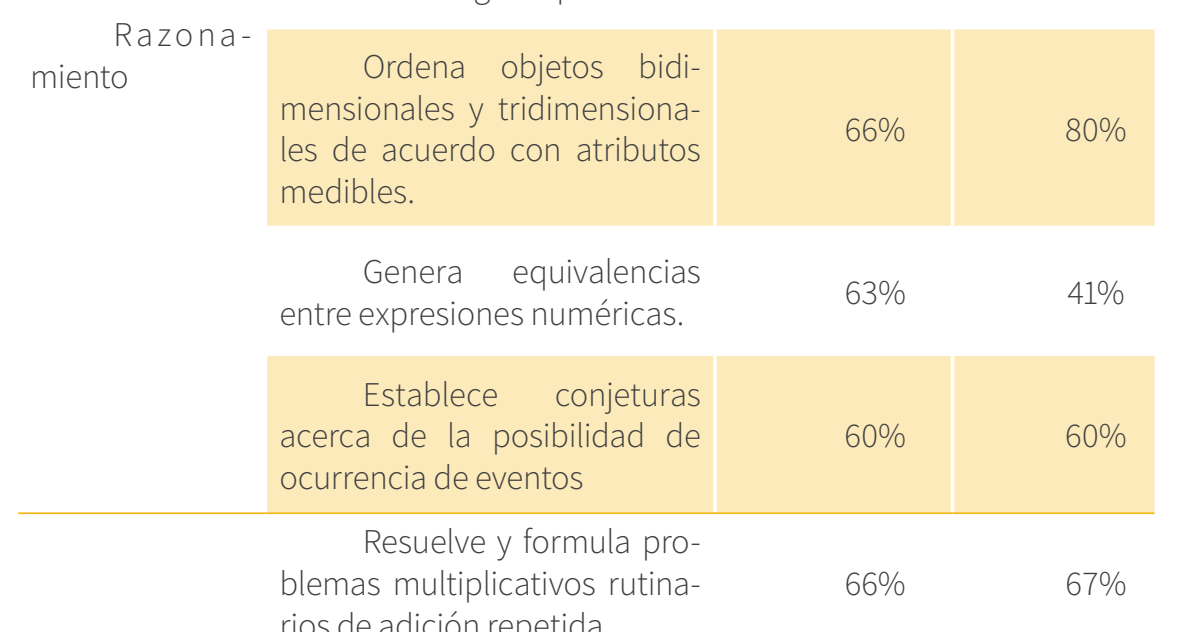
rios de adición repetida.

\begin{tabular}{|c|c|c|c|}
\hline \multirow{3}{*}{ Resolu- } & $\begin{array}{l}\text { Resuelve y formula pro- } \\
\text { blemas sencillos de proporcio- } \\
\text { nalidad directa. }\end{array}$ & $61 \%$ & $58 \%$ \\
\hline & $\begin{array}{l}\text { Resuelve situaciones } \\
\text { que requieren estimar grados } \\
\text { de posibilidad de ocurrencia } \\
\text { de eventos. }\end{array}$ & $56 \%$ & $43 \%$ \\
\hline & $\begin{array}{l}\text { Desarrolla procesos de } \\
\text { medición usando patrones e } \\
\text { instrumentos estandarizados. }\end{array}$ & $48 \%$ & $41 \%$ \\
\hline
\end{tabular}

Nota: Elaboración propia a partir del Reporte de Excelencia (ICFES, 2017)

Al comparar los resultados de las Pruebas Saber 2016 presentados por ambos cursos cuando cursaban grado tercero, se pueden realizar las siguientes interpretaciones:

$\rightarrow$ En la competencia de comunicación las dos instituciones presentaron el mismo porcentaje de dificultad (73\%), la mayor 
dificultad para ambos cursos está en la descripción de secuencias numéricas y geométricas.

$\rightarrow$ En la competencia de razonamiento se establece una diferencia del 24\%, para Institución Educativa Nuevo Compartir es la competencia con la mayor puntuación de dificultad (74\%), donde el $81 \%$ de los estudiantes no usan las operaciones y propiedades de los números para establecer relaciones entre ellos en situaciones específicas y para el Colegio Darío Echandía I.E.D se constituye como la competencia de menor puntuación (50\%), donde el ordenamiento de objetos bidimensionales y tridimensionales de acuerdo con atributos medibles presento una dificultad del 80\%.

$\rightarrow$ En la competencia de resolución se halla una diferencia del $8 \%$, para las dos instituciones es la segunda competencia con mayor dificultad, donde la mayor dificultad se presenta en la resolución y formulación de problemas multiplicativos rutinarios de adición repetida.

Queda evidenciado así que los estudiantes del curso 503 de la Institución Educativa Nuevo Compartir y 502 de la I.E.D Colegio Darío Echandía, presentaban un desempeño básico en cada una de las competencias matemáticas, sustentado tanto en las pruebas externas como internas; de ahí la importancia de elaborar una propuesta pedagógica que permita potencializar la resolución de problemas.

\section{Intervención pedagógica}

Realizada la descripción del problema, la caracterización de la población, consultados los antecedentes y un reconocimiento teórico y conceptual de la resolución de problemas matemáticas, se diseña una unidad didáctica basada en un modelo pedagógico con enfoque de aprendizaje significativo denominado: "La resolución de problemas una oportunidad para aprender".

Para Sanmartí (2005), las unidades didácticas dentro de un paradigma constructivista responden a las nuevas visiones sobre el aprendizaje, donde se considera que son los propios estudiantes quienes construyen el conocimiento mientras que la función principal de los docentes se enmarca en promover este proceso constructivo. La elaboración de unidades didácticas debe regirse por los objetivos que se persiguen teniendo en cuenta el contexto donde se planea ejecutar. Si bien un docente puede tomar un modelo ya establecido, este debe concretar, reelaborar y reinventarlo según las características de la población y sus propias consideraciones.

Sanmartí (2005), propone criterios orientadores para el diseño de una unidad didáctica; en primer lugar, se encuentra la definición de objetivos, se debe establecer la idea- matriz, entendida como la finalidad del área que se va a orientar, para ello se determina qué se considera importante enseñary cómo aprenden mejor los estudiantes. Los objetivos deben ser pocos, básicos y tener una correspondencia con el tiempo previsto de enseñanza. En segundo lugar, está la selección de contenidos los cuales se deben identificar y diferenciar de aquellos contenidos curriculares que se refieren a los conceptos, procesamientos o actitudes. Finalmente hay que organizarlos y secuenciarlos.

Para estructurar la unidad didáctica se recomienda el uso de mapas conceptuales o esquemas que pongan en manifiesto las interrelaciones de los contenidos. En la acción se propone el diseño de actividades, porque ellas posibilitan el acceso del estudiante al conocimiento, pueden responder a este orden: exploración o iniciación, aplicación, síntesis y generalización.
Por último, se debe considerar la forma de evaluación, esta debe dar cuenta del proceso de construcción del conocimiento. En general, la unidad didáctica debe estar orientada a propiciar espacios de aprendizaje donde se fomente un ambiente de clase adecuado, la comunicación asertiva, el respeto por las ideas de los demás y la elaboración de propuestas consensuadas.

La unidad didáctica "la resolución de problemas, una oportunidad para aprender", fue diseñada con el propósito de potenciar las competencias matemáticas para la resolución de problemas matemáticos en los estudiantes de quinto grado de educación básica de la Institución Educativa Nuevo Compartir y la I.E.D. Darío Echandía. Los talleres que se han diseñado guardan una correspondencia con el proceso de enseñanza propia del área, a medida que se avanza en su ejecución el nivel de complejidad aumenta. La estructura propuesta al interior de cada taller cuenta con cinco momentos, el primero de ellos es el momento de exploración, el cual consiste en el espacio donde la docente dará las indicaciones generales para la realización del taller y se hará una actividad para determinar los conocimientos previos que poseen los estudiantes sobre el tema a trabajar.

Un momento de aplicación, comprendida como la realización de la actividad central, donde el estudiante se enfrenta a problemas matemáticos desde diferentes escenarios. La conceptualización, una vez realizada la actividad se socializa, allí los estudiantes tendrán la oportunidad de justificar sus respuestas y replantear los procedimientos empleados. Un momento de evaluación, se presentan listas de chequeos y matrices para orientar el proceso de valoración del desempeño del estudiante frente a las actividades realizadas, así como su actitud y disposición. Y por último la retroalimentación, los estudiantes cuentan con un espacio para hacer el análisis de los resultados y proponer alternativas para superar las dificultades presentadas.

Para Sandoval (1996) el taller es una estrategia que permite abordar desde una perspectiva integral un fenómeno que se esté presentando en grupo social, su diseño debe partir de la caracterización de la población a la que va dirigido, se debe trazar un propósito, un tiempo de ejecución y una guía escrita preparada con antelación que oriente el proceso de aplicación. Una de las grandes ventajas de emplear esta técnica es la recolección de información sobre los avances de la intervención pedagógica dentro de un proyecto investigativo.

La unidad didáctica propuesta está conformada por diez talleres, éstos son: "Explorando mis saberes", que buscaba establecer los conocimientos previos; "Leo, identifico y comprendo" e "Interpretando ando", enfocados en fortalecer la comprensión de los enunciados de los problemas matemáticos. El cuarto taller "Resolviendo paso a paso", se realiza por grupos colaborativos, donde los niños resolvían problemas matemáticos según las fases de Polya (1965); El quinto taller "Un problema a escena", brindó el espacio para que los estudiantes representaran mediante una escena dramática un problema matemático con su solución.

"Manos a la obra" es un taller práctico donde los estudiantes armaban sólidos geométricos y a partir de su construcción, resolvían problemas matemáticos en parejas; el sexto taller, contempla un juego llamado "calcular para escalar", diseñado por el grupo investigativo con el fin de presentar a través de la lúdica la solución de problemas matemáticos. El taller "Desafío matemático" buscaba evaluar la elaboración de enunciados y realización de algoritmos a partir de una respuesta dada.

El noveno taller "Cabeza y cola" permitió que los estudiantes concertaran en grupos colaborativos los procedimientos para solucionar un problema matemático, para competir posteriormente 
La resolución de problemas, una oportunidad para aprender Intervención pedagógica en matemáticas

con otros grupos; por último, se plantea el taller "Practico lo aprendido", donde se aplica una prueba con preguntas de la Prueba Saber 2016 para grado quinto, el objetivo de esta actividad era establecer una comparación con la prueba de la actividad inicial, referente a las tres competencias resolución, comunicación y razonamiento.

\section{Resultados}

Realizadas las pruebas tipo Saber en los talleres $N^{\circ} 1$ y $N^{\circ} 10$, se relacionan los resultados en cuanto a las competencias matemáticas en las figuras 5 y 6 :

\section{Figura 5 Resultados pre test y post test Institución Educativa} Nuevo Compartir

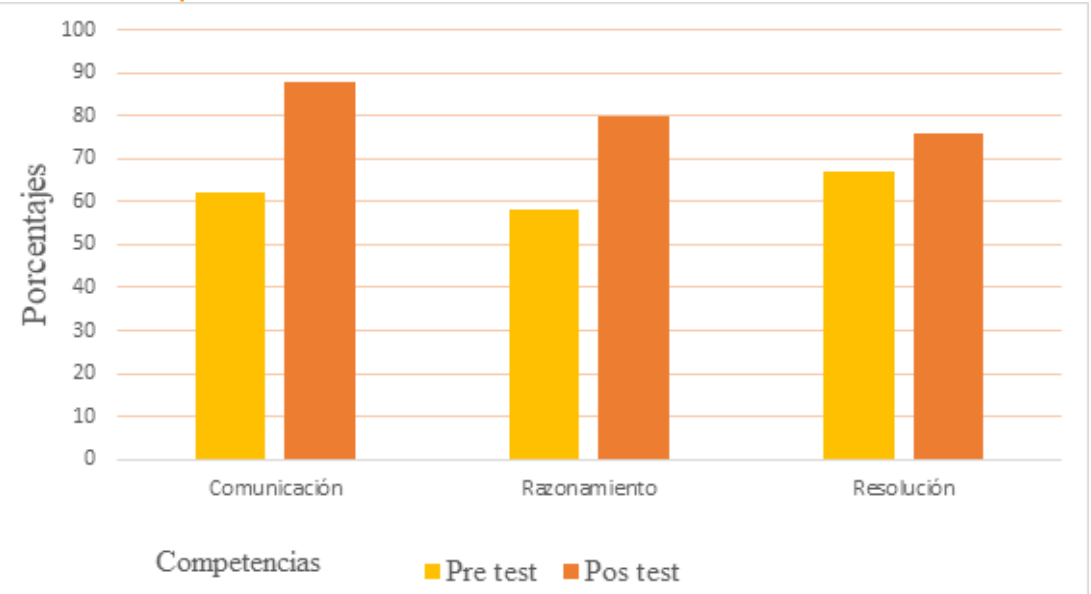

Fuente: elaboración propia a partir de los resultados de las pruebas del taller №1 "Explorando mis saberes" y №10 "Practico lo aprendido". Figura 6 Resultados pre test y post test Colegio Darío Echandía I.E.D.

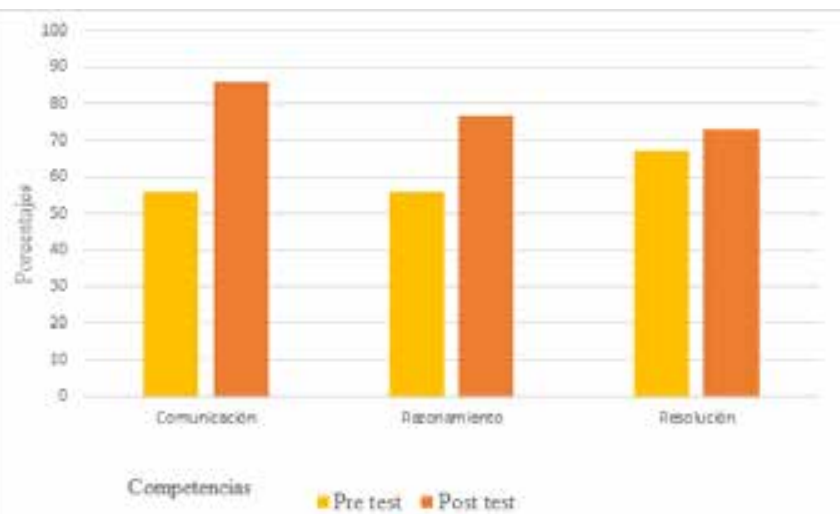

Fuente: elaboración propia a partir de los resultados de las pruebas del taller №1 "Explorando mis saberes" y N 10 "Practico lo aprendido".

Respecto a cada una de las competencias podemos señalar:

\section{Comunicación}

$\rightarrow$ La institución Educativa Nuevo Compartir presentó un aumento del $26 \%$, pasando del $62 \%$ al $88 \%$ y para la I.E.D Darío Echandía, fue del $30 \%$, donde superó del 56\% al 86\%, siendo la competencia con mayor progreso en ambas organizaciones escolares.

$\rightarrow$ Los estudiantes identifican correctamente los datos presentes en los problemas matemáticos, dedican más tiempo a la lectura de los enunciados, los interpretan en forma asertiva; ante un término desconocido preguntan por su significado, expresan ideas y situaciones que involucran conceptos matemáticos y en la socialización de los problemas emplean términos matemáticos.

\section{Razonamiento}

$\rightarrow$ La institución Educativa Nuevo Compartir presentó un aumento del $22 \%$, pasando del $58 \%$ al $80 \%$ y para la I.E.D Darío Echandía fue del $21 \%$, donde pasó del $56 \%$ al $77 \%$, siendo la competencia con el segundo puesto en progreso.

$\rightarrow$ Los estudiantes realizan diagramas de los datos presentados en los enunciados, registran en sus cuadernos los cálculos realizados, emplean medios para realizar y verificar cálculos, exploran diferentes modelos para solucionar un problema y disminuyó notablemente las preguntas sobre las operaciones que deben realizarse para solucionar el problema, en cambio adoptan una postura de disposición y concentración.

\section{Resolución}

La institución Educativa Nuevo Compartir presentó un aumento del $9 \%$, pasando del 67\% al 76\% y para la I.E.D. Darío Echandía, fue del $6 \%$, donde pasó del $67 \%$ al $73 \%$, siendo la competencia con menos progreso.

Los estudiantes elaboran un plan, justifican la solución dada a un problema atendiendo a un orden lógico, emplean términos matemáticos y resuelven problemas matemáticos derivados de situaciones cotidianas e hipotéticas.

Respecto a los pensamientos matemáticos podemos señalar los siguientes resultados en las figuras 7 y 8 :

Figura 7 Comparación de resultados por pensamientos matemáticos Pre test y Post test Institución

Educativa Nuevo Compartir

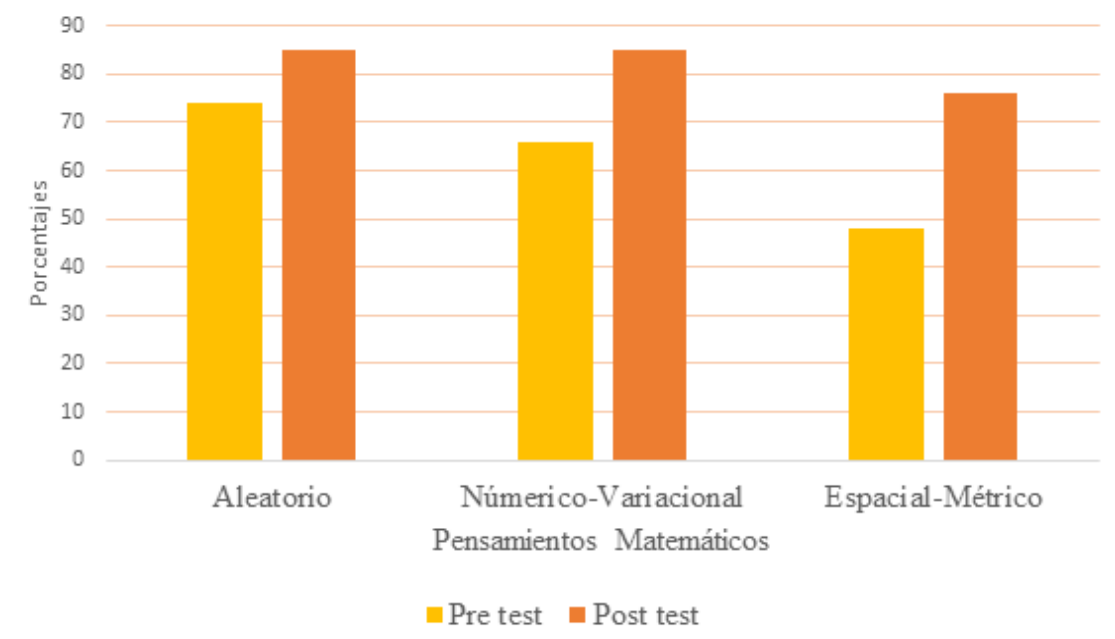

Fuente: elaboración propia a partir de los resultados de las pruebas del taller $\mathrm{N}^{\circ} 1$ "Explorando mis saberes" y N 10 "Practico lo aprendido".

Figura 8 Comparación de resultados por pensamientos matemáticos Pre test y Post test Colegio Darío Echandía I.E.D.

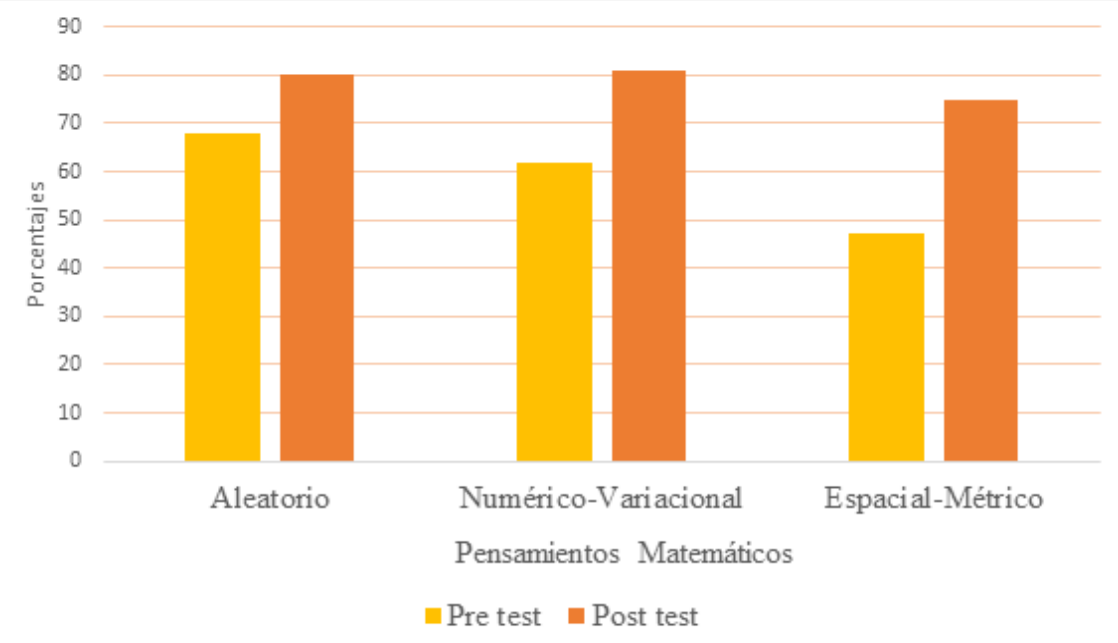

Fuente: elaboración propia a partir de los resultados de las pruebas del taller N¹ "Explorando mis saberes" y N¹0 "Practico lo aprendido".

$\rightarrow$ El pensamiento aleatorio para la Institución Educativa Nuevo Compartir presentó un aumento del 11\%, pasando del 74\% al 85\% y 
para el Colegio Darío Echandía I.E.D., fue del 12\%, pasó del 68\% al 80\%. Aunque es el pensamiento con menos progreso, continúa siendo el más puntuado, demostrando que los estudiantes de las dos instituciones educativas tienen destreza en la organización, interpretación y establecimiento de conjeturas a partir del análisis de un conjunto de datos presentados en diagramas y tablas; durante la aplicación de las dos pruebas tipo saber (taller $\mathrm{N}^{\circ} 1$ y $\mathrm{N}^{\circ} 10$ ) las respuestas con mayores aciertos pertenecían a este pensamiento.

$\rightarrow$ Los pensamientos numérico y variacional presentaron un aumento del 19\% para ambas instituciones educativas, la Institución Educativa Nuevo Compartir pasó del 66\% al 85\% y el Colegio Darío Echandía I.E.D., pasó del 62\% al 81\%; indicando un progreso en la solución de problemas matemáticos no rutinarios de comparación, combinación e igualación, en paralelo con los resultados del taller $N^{\circ} 1$.

$\rightarrow$ Los pensamientos espacial y métrico presentaron un aumento del 22\% para ambas instituciones educativas, siendo el pensamiento con el mayor progreso, pero aún se mantienen en el nivel mínimo, la Institución Educativa Nuevo Compartir transitó del 48\% al 76\% y el Colegio Darío Echandía I.E.D., pasó del 47\% al 75\%. Los estudiantes mejoraron en cuanto a la construcción y descomposición de figuras planas y sólidos a partir de condiciones dadas, pero continúan teniendo dificultades en la solución de problemas matemáticos relacionados con la medida de superficies y volúmenes.

Para hacer el análisis de los resultados de los 10 talleres que conformaron la unidad didáctica "La resolución de problemas una oportunidad para aprender", se establecieron tres categorías de análisis y se hace una confrontación con los postulados teóricos expuestos en el marco conceptual, tal y como se muestra en la tabla 4:

Tabla 4 Triangulación de la información

\begin{tabular}{|c|c|c|}
\hline Categoría & Resultados & $\begin{array}{l}\text { Relación con los } \\
\text { referentes teóricos }\end{array}$ \\
\hline $\begin{array}{l}\text { Comunicación } \\
\text { Implica conocer el } \\
\text { lenguaje propio de las } \\
\text { matemáticas. }\end{array}$ & $\begin{array}{l}\text { La institución Educativa } \\
\text { Nuevo Compartir presentó un } \\
\text { aumento del } 26 \% \text {, pasando } \\
\text { del } 62 \% \text { al } 88 \% \text { y para el } \\
\text { Colegio Darío Echandía I.E.D., } \\
\text { fue del 30\%, donde pasó del } \\
56 \% \text { al } 86 \% \text {, consolidándose } \\
\text { como la categoría con más } \\
\text { progreso durante la interven- } \\
\text { ción en ambas organizaciones } \\
\text { escolares. } \\
\text { Este progreso se ve represen- } \\
\text { tado en los estudiantes al: } \\
\text { Leer detenidamente los enun- } \\
\text { ciados de los problemas } \\
\text { Identificar los datos de los } \\
\text { problemas matemáticos } \\
\text { Interpretar en forma asertiva } \\
\text { los enunciados de problemas } \\
\text { matemáticos. } \\
\text { Preguntar por los términos } \\
\text { desconocidos. } \\
\text { Expresar ideas y situaciones } \\
\text { que involucran conceptos } \\
\text { matemáticos. }\end{array}$ & $\begin{array}{l}\text { Polya (1965), plantea como el } \\
\text { primer paso para resolver un } \\
\text { problema matemático es la } \\
\text { comprensión del enunciado, } \\
\text { lo que implica una lectura } \\
\text { detallada y una identificación } \\
\text { de los datos; Campistrous y } \\
\text { Rizo (1996), expresan que la } \\
\text { dificultad en la resolución de } \\
\text { problemas radica en la com- } \\
\text { prensión de los enunciados. } \\
\text { Yal ser la comunicación, la } \\
\text { competencia más puntuada } \\
\text { demuestra que los estudian- } \\
\text { tes de grado quinto de ambas } \\
\text { instituciones educativas se } \\
\text { encuentran en un proceso } \\
\text { de potencialización de la } \\
\text { resolución de problemas } \\
\text { matemáticos. }\end{array}$ \\
\hline
\end{tabular}

\begin{tabular}{|c|c|c|}
\hline Categoría & Resultados & $\begin{array}{l}\text { Relación con los } \\
\text { referentes teóricos }\end{array}$ \\
\hline $\begin{array}{l}\text { Razonamiento } \\
\text { Capacidad que tiene el } \\
\text { estudiante para anali- } \\
\text { zar, argumentary pla- } \\
\text { near una estrategia para } \\
\text { resolver un problema. }\end{array}$ & $\begin{array}{l}\text { La institución Educativa } \\
\text { Nuevo Compartir presentó un } \\
\text { aumento del 22\%, pasando } \\
\text { del } 58 \% \text { al } 80 \% \text { y para el Cole- } \\
\text { gio Darío Echandía I.E.D., fue } \\
\text { del 21\%, donde pasó del } 56 \% \\
\text { al } 77 \% \text {, siendo la competencia } \\
\text { con el segundo puesto en } \\
\text { progreso, evidenciándose al: } \\
\text { Realizar diagramas y dibujos } \\
\text { para representar la informa- } \\
\text { ción de los enunciados. } \\
\text { Explorar diferentes modelos } \\
\text { para solucionar un problema. } \\
\text { Emplear medios para realizar } \\
\text { y verificar cálculos. } \\
\text { Disminuyó las preguntas } \\
\text { de los estudiantes sobre el } \\
\text { algoritmo que debe realizarse } \\
\text { para solucionar el problema. }\end{array}$ & $\begin{array}{l}\text { Para Zabala (2007), la difi- } \\
\text { cultad para solucionar un } \\
\text { problema matemático radica } \\
\text { en la selección de un esque- } \\
\text { ma que pueda darle solución; } \\
\text { Polya (1965), contempla } \\
\text { la elaboración de un plan } \\
\text { como el segundo paso para } \\
\text { abordar una situación ma- } \\
\text { temática. El avance en esta } \\
\text { categoría se fue evidenciando } \\
\text { en la medida que los estu- } \\
\text { diantes realizaban diagramas } \\
\text { para representar los datos del } \\
\text { problema, establecían mode- } \\
\text { los de solución y realizaban } \\
\text { cálculos en el cuaderno. }\end{array}$ \\
\hline $\begin{array}{l}\text { Resolución } \\
\text { Capacidad que tiene } \\
\text { el estudiante para } \\
\text { enfrentarse a proble- } \\
\text { mas matemáticos no } \\
\text { rutinarios que requieren } \\
\text { de nuevos modelos } \\
\text { para resolverse. }\end{array}$ & $\begin{array}{l}\text { La institución Educativa } \\
\text { Nuevo Compartir presentó un } \\
\text { aumento del 9\%, pasando del } \\
67 \% \text { al } 76 \% \text { y para el Colegio } \\
\text { Darío Echandía I.E.D., fue del } \\
\text { 6\%, donde pasó del } 67 \% \text { al } \\
73 \% \text {, siendo la competencia } \\
\text { con menos progreso entre } \\
\text { las tres categorías de análisis. } \\
\text { Los avances en esta compe- } \\
\text { tencia se ven presentes en los } \\
\text { estudiantes al: } \\
\text { - Elaborar y ejecutar un } \\
\text { plan para resolver un proble- } \\
\text { ma matemático. } \\
\text { - Resolver problemas } \\
\text { matemáticos derivados de } \\
\text { situaciones cotidianasy } \\
\text { matemáticas. }\end{array}$ & $\begin{array}{l}\text { El MEN (2006), define la com- } \\
\text { petencia de resolución como } \\
\text { la capacidad que tiene implí- } \\
\text { cita las demás competencias, } \\
\text { porque requiere de varias } \\
\text { habilidades matemáticas } \\
\text { que le permitan al estudiante } \\
\text { enfrentarse a situaciones } \\
\text { problemas no rutinarias, } \\
\text { por ende, esta categoría de } \\
\text { análisis presenta el progreso } \\
\text { más bajo, porque necesita } \\
\text { más tiempo y esfuerzo para } \\
\text { potenciarse. }\end{array}$ \\
\hline
\end{tabular}
los teóricos referenciados en el marco conceptual.

Para medir el impacto de la unidad didáctica se establecieron dos instrumentos, grupos de discusión entre los estudiantes de las dos instituciones educativas, un taller de grupo focal con los padres de familia y se hizo un análisis de los informes académicos del segundo periodo; los resultados fueron los siguientes:

$\rightarrow$ Grupos de discusión: a los estudiantes de la Institución Educativa Nuevo Compartir se les asignó un estudiante del Colegio Darío Echandía I.E.D., a los cuales les redactaron una carta donde contaban su experiencia en la Unidad Didáctica "La resolución de problemas una oportunidad para aprender", resaltando aspectos acerca del taller de preferencia, dificultades presentadas, incidencia de los talleres en el desempeño en el área de matemáticas e invitaban a sus compañeros a contar su experiencia. Por su parte, los estudiantes del colegio Darío Echandía respondieron las cartas, tratando los mismos puntos. Las cartas se enmarcaron bajo la misma estructura: fecha, ciudad, nombre del estudiante al cual iba dirigida la carta, asunto, saludo, narración de la experiencia y datos del remitente.

Realizada la lectura y el análisis de las cartas se puede señalar que los talleres que fueron más significativos para los estudiantes son aquellos que propician su participación activa a través del juego y el trabajo en equipo, presentarles problemas matemáticos en diferentes 
La resolución de problemas, una oportunidad para aprender Intervención pedagógica en matemáticas

escenarios les permite hacer uso de sus habilidades (artísticas, sociales y comunicativas) en función de un logro académico, llegando a reconocer y valorar el impacto de los talleres en el desempeño en el área de matemáticas y en las relaciones interpersonales en el salón de clases.

$\rightarrow$ Grupos focales: Durante la reunión de padres de familia se conformaron los grupos con las dos instituciones educativas, a quienes se les entregó una guía de trabajo para evaluar el impacto de la unidad didáctica donde se les pedía responder cinco preguntas orientadas a indagar sobre el conocimiento que tenían los padres de familia respecto a los objetivos, los talleres aplicados, el aporte al rendimiento académico y disciplinario de los estudiantes, además se brindó el espacio para las observaciones y sugerencias.

Respecto a las respuestas se puede destacar que un 75\% de los papas señalaron como objetivo de la unidad didáctica aprender a resolver problemas matemáticos y un 25\% complementaron la respuesta indicando el medio, en las respuestas se evidencia claridad sobre el tema de la unidad didáctica y de los medios empleados para alcanzar el objetivo. Los talleres más mencionados por los padres de familia son "Cabeza y cola", "Calcular para escalar", "Un problema a escena” y "Manos a la obra", estas respuestas coinciden con las dadas por los niños en los grupos de discusión, confirmando que los talleres más significativos son aquellos donde pueden participar activamente, interactuar con sus compañeros y realizar actividades que están relacionadas con sus propios intereses.

Los padres de familia señalan que los talleres aplicados incidieron significativamente en su desempeño en el área de matemáticas, mejorando la disposición de los estudiantes en el proceso de aprendizaje, se muestran más entusiastas por asistir al colegio, comparten con sus hermanos y demás familiares las experiencias vividas en la aplicación de los talleres y disminuyeron significativamente los llamados de atención por el mal comportamiento durante las clases. Como observaciones los padres de forma unánime señalaron la importancia de aplicar la unidad didáctica en otros niveles escolares, trascender a otras áreas del conocimiento y que fueran involucrados en el proceso.

$\rightarrow$ Informes académicos del primer y segundo periodo: En las figuras 9 y 10 se muestra el desempeño de los estudiantes en el área de matemáticas de las dos instituciones educativas, haciendo un comparativo.

Figura 9 Informe académico primer periodo vs segundo periodo, Institución Educativa Nuevo Compartir.

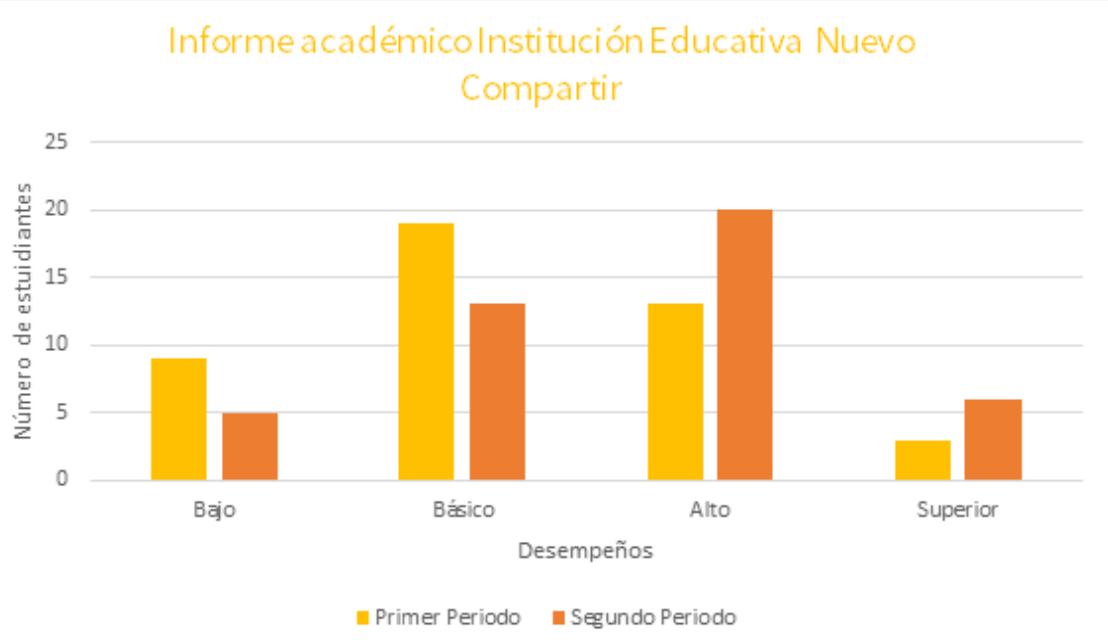

Fuente: elaboración propia a partir del registro de notas del segundo periodo en el área de matemáticas.
Figura 10 Informe académico, primer periodo vs segundo periodo Colegio Darío Echandía I.E.D.

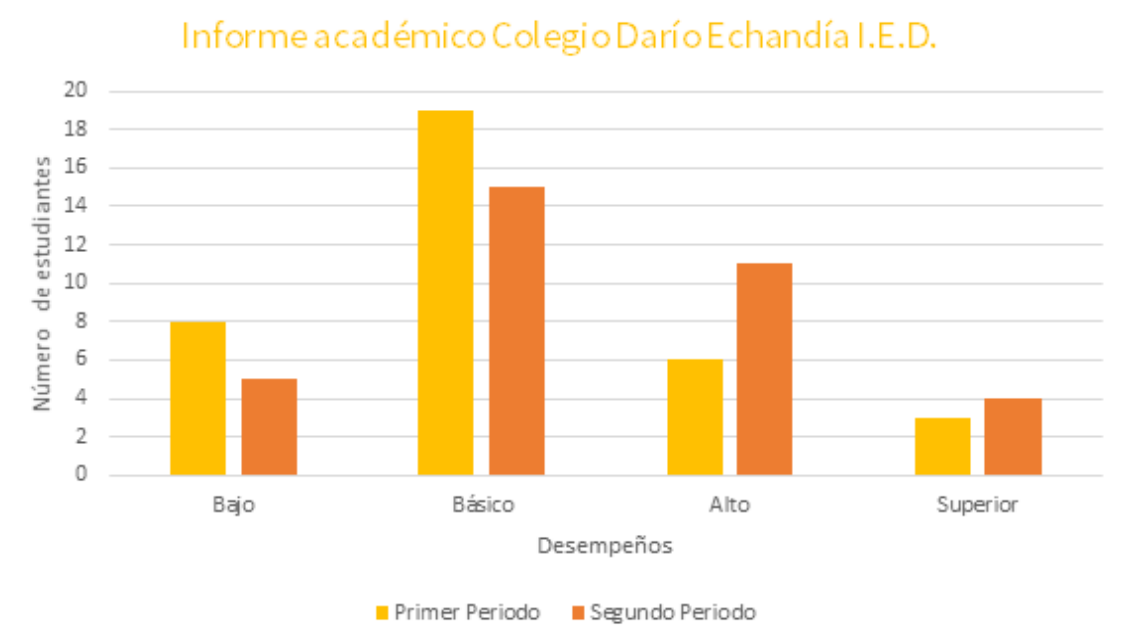

Fuente: elaboración propia a partir del registro de notas del segundo periodo en el área de matemáticas.

El desempeño en el área de matemáticas para los grados quinto de las dos instituciones educativas ha presentado un gran avance, representado en la disminución de los estudiantes con desempeño bajo, para la Institución Educativa Nuevo Compartir, la tasa de reprobación pasó del 20\% al $11 \%$, los desempeños alto y superior pasaron de un 36\% al 59\%, con un incremento del 23\%; para el Colegio Darío Echandía I.E.D., la tasa de reprobación bajó, pasó del 22\% al 14\%, los desempeños alto y superior pasaron del 25\% al 42\% con un incremento del $17 \%$. De esta manera la incidencia de la aplicación de los talleres también queda demostrado en las calificaciones, convirtiéndose en un gran incentivo tanto para los estudiantes como para los padres de familia al evidenciar en forma concreta sus logros.

Recopiladas las experiencias de los estudiantes y las percepciones de los padres de familia de las dos instituciones educativas, se puede señalar el impacto de la Unidad Didáctica y su aplicabilidad en otros contextos educativos, convirtiéndose en una estrategia pedagógica que potencia las competencias matemáticas para la resolución de problemas matemáticos en los estudiantes de quinto grado de educación básica.

\section{Conclusiones}

Realizado el proyecto de investigación se pueden señalar 8 conclusiones principales:

$\rightarrow$ La implementación de la unidad didáctica, permitió potenciar las competencias matemáticas para la solución de problemas matemáticos, aunque cabe señalar que la competencia de resolución se encuentra en un nivel básico, no obstante, los estudiantes ya tienen incorporado el proceso para solucionar un problema la lectura detallada del enunciado, la identificación de datos, la representación gráfica y el uso de terminología clave para la elaboración de un plan.

$\rightarrow$ Al socializar el proceso para solucionar un problema matemático, los estudiantes hacen uso de conceptos matemáticos, referencian los cálculos realizados y dan un orden lógico a los procedimientos, consolidando argumentos claros.

$\rightarrow$ La realización del taller Nº "Manos a la obra”, permite afirmar que para lograr que los estudiantes lleguen a la composición y descomposición de figuras geométricas, se debe abordar primero desde las aulas el trabajo con material concreto, ya que este afirmará los procesos de construcción, afianzará los términos y permitirá al estudiante corroborar el plan trazado. 
$\rightarrow$ Los problemas matemáticos que más se les facilita a los estudiantes son los relacionados con la interpretación de datos presentes en tablas y gráficos estadísticos, siendo los problemas con mayor puntuación durante la realización de las pruebas de los talleres $N^{\circ} 1$ "Explorando mis saberes" y N¹0 "Practico lo aprendido".

$\rightarrow$ Cuando se les pide resolver un problema matemático, los estudiantes se concentran en la actividad, realizan cálculos en el cuaderno y preguntan por los términos desconocidos; se debe destacar que las preguntas relacionadas con el algoritmo o proceso que debe emplearse disminuyeron notablemente, demostrando una disposición favorable para trabajar la solución de problemas matemáticos.

$\rightarrow$ Los resultados obtenidos en la aplicación de cada uno de los talleres demuestran que los estudiantes mejoraron en cuanto a la identificación de los datos, de términos claves y en la selección del procedimiento, esto se debe a los espacios que se han ofrecido a lo largo del proyecto para la solución de problemas; tal como lo afirma Polya (1965), resolver problemas es una cuestión de habilidad práctica, para lo cual se debe ejercitar al estudiante, la tarea del docente consistirá en proporcionarle el mayor número de ocasiones de imitación y práctica.

$\rightarrow$ Al trabajar problemas matemáticos relacionados con el contexto de los estudiantes, se propició el espacio para que éstos comprendieran el uso y aplicabilidad de los algoritmos y procedimientos matemáticos en la vida real; siendo significativos los aprendizajes generados durante la implementación de la unidad didáctica.

$\rightarrow$ Los talleres "Interpretando ando", "Resolviendo paso a paso", "Calcular para escalar", "Un problema a escena” y "Cabeza y cola" que propiciaban el trabajo en grupos colaborativos, fomentaron habilidades sociales como el respeto por la opinión del otro, la tolerancia, el trabajo conjunto y la cooperación; favoreciendo así las relaciones entre pares y el ambiente escolar.

Por el impacto de la unidad didáctica "La resolución de problemas una oportunidad para aprender" en las dos instituciones educativas, evidenciados en los resultados del pre test y post test, las experiencias de los estudiantes y las percepciones de los padres de familia, se puede señalar que es una estrategia pedagógica aplicable en otros contextos educativos, que potencia las competencias matemáticas para la resolución de problemas matemáticos en los estudiantes de quinto grado de educación básica.

A partir del trabajo investigativo se pueden señalar las siguientes recomendaciones:

$\rightarrow$ Al redactar los enunciados de los problemas matemáticos es importante que estén relacionados con el contexto de los estudiantes, así descubrirán por sí mismos la utilidad de los algoritmos matemáticos en situaciones cotidianas; en este sentido Ausubel (1978), nos dice que todo conocimiento adquirido llevado a la práctica en un contexto real cobra sentido y es significativo.

$\rightarrow$ Para potenciar la resolución de problemas se le debe proporcionar al estudiante el mayor número de ejercitaciones en diferentes escenarios; Polya (1965), sostiene que resolver problemas es una cuestión de habilidad práctica, para lo cual se debe ejercitar al estudiante, la tarea del docente consistirá en proporcionarle el mayor número de ocasiones de imitación y práctica, fortaleciendo así las competencias necesarias para solucionar asertivamente situaciones matemáticas.

$\rightarrow$ Siendo la comprensión de los enunciados el primer paso para resolver situaciones matemáticas se convierte en el primer punto a tratar, por ende, un plan encaminado hacia la resolución de problemas debe considerar espacios para la identificación de datos, lectura comprensiva de los enunciados, determinación de la incógnita y proyección de la respuesta, tal y como lo afirman Campistrous L. y Rizo C. (1996), la dificultad en la resolución de problemas radica en la comprensión lectora, por eso la escuela debe proporcionar técnicas como la modelación, la lectura analítica, la reformulación y comprobación.

$\rightarrow$ Una de las principales dificultades en la enseñanza de la resolución de problemas para Hezkuntza (2000), es la transferencia de los conocimientos matemáticos a los contextos o situaciones, por consiguiente, la estrategia de intervención que el docente elabore debe estar basada en la localización de centros de interés, el trabajo cooperativo, la autonomía y la participación activa del estudiante.

$\rightarrow$ El trabajo por grupos colaborativos y en parejas permite que los estudiantes desempeñen diferentes roles, fortalezcan sus habilidades sociales como saber escuchar, respetar los turnos de participación, expresar en forma adecuada un punto de vista, la empatía y las competencias necesarias para la solución de problemas (comunicación, razonamiento y resolución); para Vygotski (1934), el trabajo en pares posibilita la zona de desarrollo próximo (ZDP) conocida como la brecha o diferencia entre las habilidades y destrezas que ya posee el estudiante y lo que puede llegar a aprender a través de la guía o mediación que le puede proporcionar un adulto o un par más competente.

$\Rightarrow$ En el campo de las matemáticas se han realizado múltiples investigaciones, entre las que se pueden destacar: Navarro (2016) "Función de la memoria de trabajo en diferentes programas de habilidades matemáticas", como resultado el grupo experimental mejoró la memoria de trabajo verbal y visoespacial, además aumentó la precisión en la realización de adiciones y sustracciones; Granados y Rodríguez (2011) “Implementación de un procedimiento generalizado para la resolución de problemas en el área de matemáticas en básica primaria" donde se plantea un procedimiento en 3 fases: orientación, ejecución y fase de constatar donde se comprueba las operaciones realizadas; Campos y Devia (2013) "Desarrollo de las competencias de razonamiento y argumentación en estudiantes de $5^{\circ}$ de educación básica primaria", como propuesta plantean la resolución de problemas como eje dinamizador de los procesos de enseñanza y aprendizaje de las matemáticas para favorecer el desarrollo de las competencias; Rodríguez (2015) "El desarrollo de competencias matemáticas a través de las tareas de investigación en el aula", como logros dentro de la investigación se señala que los estudiantes hacen uso del lenguaje matemático, diseñan procedimientos basados en esquemas personales, hacen una organización y registro correcto de datos.

Respecto a estas propuestas para intervenir las dificultades en la resolución de problemas se coincide en el uso de estrategias pedagógicas basadas en los intereses de los estudiantes, relacionadas con su contexto, flexibles, dinámicas, que propician la participación activa y deslumbren el uso de los conocimientos matemáticos en situaciones de la vida real. Por ello, la propuesta pedagógica "La resolución de problemas una oportunidad para aprender" toma los aspectos anteriormente expuestos y se basa en un modelo pedagógico constructivista, para Sanmartí (2005), la implementación de este tipo de unidades permite que el estudiante construya su conocimiento, mientras que el papel del docente se enfoca en promover ese proceso constructivo. Además, la unidad didáctica está conformada por diez talleres que varían su nivel de complejidad, para dar cuenta del progreso que van presentando los estudiantes en la resolución de problemas matemáticos y las actividades planteadas priorizan la comprensión de los enunciados y la identificación de datos, factores determinantes en la solución de problemas matemáticos. 
La resolución de problemas, una oportunidad para aprender Intervención pedagógica en matemáticas

$\rightarrow$ Por el impacto de la unidad didáctica "La resolución de problemas una oportunidad para aprender" en las dos instituciones educativas, la convierte en una estrategia pedagógica aplicable en otros contextos; para su aplicación el docente debe conocer y tener la habilidad en la resolución de problemas, hacer un diagnóstico para establecer el nivel en que se encuentra las competencias de comunicación, razonamiento y resolución y, adaptar los enunciados de los problemas matemáticos para que respondan al contexto donde se desenvuelven los estudiantes, así se sentirán identificados, reconocerán la aplicabilidad de los procedimientos matemáticos y llegarán al aprendizaje significativo.

$\Rightarrow$ Para próximas investigaciones sobre la resolución de problemas matemáticos se sugiere considerar algunos ejes temáticos tales como: el trabajo cooperativo como estrategia pedagógica en la resolución de problemas, las pruebas estandarizadas como instrumento de evaluación de las competencias matemáticas y las unidades didácticas bajo un modelo constructivista como herramienta de intervención en la resolución de problemas matemáticos.

\section{Referencias}

Ausubel, D., Novak, J., \& Hanesian, H. (1978). Educational Psychology: A Cognitive View [Psicología educativa: una visión cognitiva]. New York: Holt, Rinehart \& Winnston.

Campistrous, L., \& Rizo, C. (1996). Aprender a resolver problemas matemáticos. Habana: Pueblo.

Campos, E., \& Devia, C. (2013). Desarrollo de la competencia de razonamiento y argumentación de estudiantes de quinto de básica primaria. Escenarios, 87-97.

Colegio Darío Echandía I.E.D. (2017). Manual de Convivencia. Bogotá.

Corbetta, P. (2007). Metodologías y Técnicas de Investigación Social. Madrid: Mc Graw Hill.
Granados, C., \& Rodríguez, F. (2011). Implementación de un proceso generalizado para la resolución de problemas en el área de matemáticas en básica primaria. Escenarios, 37-45.

Guzman, M. (1989). Tendencias actuales de la enseñanza de la matemática. Revista de Ciencias de la Educación, 19-26.

Hezkuntza. (2000). Competencia matemática. Vasco: Departamento de Educación Universitaria e Investigación.

ICFES. (2015). Cuadernillo Prueba Saber. Bogotá: ICFES.

ICFES. (2016). Cuadernillo Prueba Saber. Bogotá: ICFES.

ICFES. (2016). Reporte de Excelencia. Bogotá: ICFES.

Institución Educativa Nuevo Compartir. (2017). Manual de Convivencia 2017. Soacha: Institución Educativa Nuevo Compartir.

Ministerio de Educación Nacional. (2016). Derechos Básicos de Aprendizaje. Bogotá: MEN.

Ministerio de Educadión Nacional. (2006). Estándares Básicos de Matemáticas. En MEN, Estándares Básicos de Matemáticas (pág. 42) Colombia: MEN.

Navarro, M. (2016). La función de la memoria de trabajo en diferentes programas de habilidades matemáticas. Cadíz: Universidad de Cadíz.

Perrenound, P. (2001). La formación de docentes en el siglo XXI. Revista Tecnológica Educativa, 503-523.

Polya. (1965). Cómo plantear y resolver problemas. México: Trillas.

Pozo, J., \& M, G. (1995). Aprendizaje de estrategias para la solución de problemas en ciencias. Madrid: Alambique.

Rodríguez, F. (2015). El desarrollo de competencias matemáticas. Madrid: UNED.

Sandoval, C. (1996). Investigación Cualitativa. Bogotá: ICFES.

Sanmartí, N. (2005). La Unidad Didáctica en el paradigma constructivista, capítulo 1. Unidades didácticas en ciencias y matemáticas. Bogotá: Magisterio.

Zabala. (2007). Cómo aprender y enseñar competencias. Barcelona: GRAÓ. 